\title{
Hypnerotomachia Poliphili - per un sincretismo culturale
}

Il Quattrocento, come ogni epoca, tende a trovare un proprio modo di descrivere l'universo, conforme ai contesti storico-culturali da cui nasce e adeguato all'immaginario individuale e collettivo in cui si forma. Se per indicare un peculiare significato dell'umanesimo italiano, vogliamo partire dagli studi di Eugenio Garin, ci troviamo davanti a un'interpretazione spazio-temporale dell'epoca che vede i suoi meriti in "un dialogo interumano fuori spazio e fuori tempo" e con ciò dichiara di non riconoscere limitazioni al pensiero e all'agire umano, affermando, invece, la possibilità, anzi, la necessità di oltrepassarne i confini.

Il capolavoro di Francesco Colonna, in primis, parla dell'unione di spazi separati tra realtà contigue e appartenenti alla stessa cultura o a culture differenti che si riconoscono a vicenda e, in secundis, si riferisce alle dimensioni dello spazio conosciuto e del mondo noto, in quanto delimita terreni, luoghi o aree geografiche realmente esistenti. Accanto ai confini spaziali che indicano le estremità di territori, edifici, oggetti e strutture architettoniche, un loro ruolo acquistano i confini temporali che testimoniano una consapevole coscienza della fine di un'epoca e del passaggio a un'altra, concetti che Colonna unisce con quelli legati alla sfera ideologica e religiosa. Siamo davanti a un'opera che secondo intentio auctoris può essere letta in modi diversi e a svariati livelli: come 
storia d'amore, come guida archeologica fra le rovine del passato, come viaggio iniziatico, come manuale di filosofia quattrocentesca, per indicarne solo alcuni: infatti, nel testo c'è tutto quello che ha da trasmettere l'umanesimo italiano. Dietro le vicende del romanzo, che a livello narrativo si mostrano abbastanza facili da sintetizzare, si nascondono più profondi significati filosofici e culturali, che trovano la loro migliore manifestazione nelle tendenze che si prestano molto bene all'analisi della visione di Francesco Colonna in chiave sincretistica, caratteristica per l'area mediterranea e mediorientale.

In questo contributo mi propongo di riflettere sulla lettura del capolavoro di Francesco Colonna, partendo dalle nozioni topologiche in quanto espedienti della descrizione dell'universo umano e cosmico che determinano l'idea del sincretismo, inteso come conciliazione di diverse esperienze culturali, intellettuali e religiose ed espresso dalla trasgressione di confini e limiti. ${ }^{1}$ In seguito, vorrei soffermarmi sulle possibilità interpretative che esse offrono e proporre un percorso sulla scia delle tavole illustrative a tutta pagina intercalate nel testo. Di particolare interesse saranno i significati comportati dalle prime due silografie a piena pagina e dall'ultima, in quanto si riferiscono ai punti estremi della fase iniziale e di quella finale del viaggio dell'eroe e trasmettono messaggi cruciali dell'umanesimo italiano sulla simbolica natura delle cose, in grado di svelare i legami tra la materia e l'idea, la realtà e l'arte e, anzitutto, tra culture differenti, lontane nello spazio e nel tempo.

Alla base di diversi modelli di concettualizzazione del mondo e dei fenomeni presenti negli spazi simbolici sta la topologia con le sue nozioni e i suoi oggetti che permettono di indicare analogie e somiglianze indirette tra svariati testi di cultura. La topologia, infatti, nel senso generale di spazio, si presta molto bene a un approccio elementare all'analisi della visione di Francesco Colonna che la trasmette ricorrendo alle elementari nozioni matematiche e letterarie in grado di esprimere le somme capacità della mens humana. Lo studio delle forme stabili e universali trova la sua migliore realizzazione in quella branca del sapere che si fonda sui concetti come limite, connessione, convergenza,

Sull'aspetto religioso del sincretismo, cfr. R. Pettazzoni, Sincretismo e conversione nella storia delle religioni, "Bulletin du Comité international des sciences historiques", 18, 1933, pp. 24-31. 
continuità. Se l'Hypnerotomachia è una proposta filosofico-sincretistica in cui appaiono elementi della topologia e dell'aritmetica dell'ordine, contrapposte a quelle del caos e dell'emergenza, vanno esaminate le funzioni del limes e del finis, in quanto espedienti della descrizione dell'universo umano e cosmico dell'autore che nelle quantità, nei numeri, nelle strutture e nei calcoli vede una forma di cognizione dell'universo non solo cosmologico, ma anche storicoculturale. Susseguiranno i metaforici confini dell'anima che, come diceva Eraclito, non si riescono a trovare: "Per quanto tu possa camminare, e neppure percorrendo intera la via, tu potresti mai trovare i confini dell'anima: così profondo è il suo logos". ${ }^{2}$

Quali sono i contesti e i modi, in cui il confine e il limite edificano, passo per passo, il mondo nel romanzo del Colonna? Nel viaggio che compie Polifilo per ricongiungersi con la sua amata Polia non esistono i confini temporali: gli elementi dominanti della realtà rappresentata prendono le forme di edifici e di oggetti che con il loro aspetto richiamano gli antichi mondi, egizio e greco-romano e, al tempo stesso, il mondo dell'Italia quattrocentesca; è un'evocazione del passato e del contemporaneo, sempre in riferimento a tutto il sapere umano sull'arte e sul pensiero dei paesi lontani, sulle loro culture e credenze. Gli elementi della realtà rappresentata coesistono con la poetica del sogno dominata da svariate strutture e raffigurazioni; l'immaginazione creativa attinge qui alla conoscenza dei principi dell'architettura, ma le fascinazioni architettoniche non hanno limiti, perché nel mondo onirico può accadere tutto e possono esistere ogni sostanza e ogni forma. Le costruzioni e le forme descritte nell'Hypnerotomachia, che sono irreali, ma potrebbero esistere in realtà, nello spazio narrativo segnano le tappe del viaggio dell'eroe. Mentre il viaggio avanza, permettono di svelare e chiarire le successive questioni della filosofia di conoscenza, allargando l'orizzonte del sapere sulle cose del mondo e dell'uomo. Le singole fasi del percorso di Polifilo sono delimitate da diverse costruzioni e vari elementi architettonici: la strada conduce dall'oggetto all'oggetto e ciascuno di essi è una rappresentazione figurativa di un problema filosofico o di un'impronta culturale. Uno dei primi edifici che incontra Polifilo è una struttura ibrida i cui singoli componenti appartengono a culture diverse: greca, latina e quella egizia in particolare. In parte tempio, in

2 D.K. 22 B 45. 
particolare propileo, in parte piramide ed in parte obelisco, la struttura rappresenta l'unione dei mondi distanti nello spazio e nel tempo. La dettagliata descrizione della piramide è accompagnata dall'illustrazione silografica che rappresenta la costruzione nella sua dimensione completa e nella sua piena bellezza. ${ }^{3}$ E una delle prime illustrazioni del libro, che ne offre ben centosettantadue, la quinta in generale e la prima a tutta pagina e a figura isolata. ${ }^{4}$ L'armonia fra il testo e l'immagine non è una semplice conseguenza della capacità decorativa in rapporto alla pagina, o facoltà calcolativa utilizzata per guadagnare spazio nel corpo del libro, ma il risultato di una raffinata tecnica e strategia di narrazione iconico-testuale tesa a raggiungere una perfetta complementarietà tra la parola e la figura. Considerati la tipologia e i modi della disposizione delle illustrazioni nel libro, si osserva che le figure a piena pagina sono dodici, numero di forte significato simbolico, che rispecchia un modello cosmico di pienezza e di armonia ed esprime la conclusione di un ciclo compiuto. Le silografie a tutta pagina sono distribuite nel modo seguente:

$\mathrm{b} v$ : piramide.

b vii $v$ : elefante.

c viii $r$ : porta del tempio.

f $v$ : fontana.

m vi $r$ : Priapo con la falce.

$\mathrm{n}$ iii $r$ : "miraveglioso templo".

q v $v$ : antico epitaffio nel "destructo tempio".

q viii $r$ : antico epitaffio nel "destructo tempio".

$\mathrm{r}$ ii $r$ : struttura sepolcrale con le ceneri della regina Artemisia.

r iii $r$ : sepolcro con due busti.

$\mathrm{r}$ iiii $r$ : sepolcro con un'arca con due porticine.

F iii $v$ : "epitaphium Poliae" ed "epitaph. ubi Pol.loquitur".

F. Colonna, Hypnerotomachia Poliphili, a cura di M. Ariani-M. Gabriele, Milano, Adelphi, 2004, vol. I, p. b $v$. I volumi di riferimento in questo articolo sono: per la riproduzione del testo aldino del 1499 - HP, vol. I; per la tr. it. - ivi, vol. II.

4 Sull'Incisione cfr. C.A. Petrucci-M. Pittaluga, Enciclopedia Italiana di scienze, lettere ed arti, 1933, vol. XVIII; [on-line:] [http://www.treccani.it/enciclopedia/incisione_ \%28Enciclopedia-Italiana\%29/] - 12 XI 2020. 
Le prime due illustrazioni a piena pagina sopracitate - piramide (b i $v$ ) ed elefante (b vii $v)^{5}$ - si riferiscono al termine della fase iniziale del viaggio di Polifilo che si ritrova "in una convalle, la quale nel fine era serata de una mirabile clausura cum una portentosa pyramide de admiratione digna, et uno excelso obelisco de sopra". ${ }^{6}$ Quell'enorme oggetto architettonico che costituisce il primo punto nodale nel percorso dell'eroe, unisce i due simboli della cultura orientale: la piramide e l'obelisco. La costruzione è testimone delle culture antiche e l'espressione dell'interesse degli umanisti verso l'Oriente e le cose egiziane in particolare ${ }^{7}$ e, come leggiamo negli scritti ficiniani: “[...] Ægyptiis praefuisse, eisque leges ac literas tradisse, literarum vero characteres in animalium, arborumque figuris instituisse". ${ }^{8}$

Le monumentali costruzioni egiziane non hanno eguali in ambito occidentale; l'obelisco, insieme con le piramidi, è un tipo di simulacra Ægipti: istoriato da geroglifici è trasmettitore dei segni ieratici, della scrittura dei sacerdoti, alla quale deve essere consegnata l'antica sapienza. ${ }^{9}$ Nondimeno, la funzione dell'obelisco polifilesco non è religiosa: l'oggetto, attraverso segni antichi, nelle lingue del passato e del presente esprime dei concetti universali e costituisce il finis di una tappa iniziatica nel percorso di chi vuole scoprire e determinare il proprio posto nell'universo. Nell'Hypnerotomachia l'obelisco e la piramide si uniscono in una struttura singola e diventano un oggetto unico, simbolo dell'unione dei significati che porta ognuna delle dette costruzioni. Tale complesso e bizzarro edificio rappresenta la somma del sapere e dell'arte di paesi antichissimi, considerati culla della conoscenza e terra di

\footnotetext{
$5 \quad$ Per la riproduzione delle xilografie ( $\mathrm{b}$ i $v$ ) e (b vii $v$ ) v. rispettivamente la fig. 12 e la fig. 14 nel testo di N. Koch in questo volume. Anche la fig. 7 e la fig. 13 nella parte "TAVOLE".

$H P$ a vi $v$.

Cfr. P. Castelli, I geroglifici e il mito dell'Egitto nel Rinascimento, Firenze, Olschki, 1979, p. 7. Sui geroglifici nell'Hypnerotomachia v. K. GieHlow, Die Hieroglyphenkunde des Humanismus in der Allegorie der Renaissance besonders der Ehrenpforte Kaisers Maximilian I, WienLeipzig, Tempsky, 1915; K. GieHLow, The Humanist Interpretation of Hieroglyphs in the Allegorical Studies of the Renaissance. With a Focus on the Triumphal Arch of Maximilian I, tr. ingl. R. RAYвоuld, Leiden, E.J. Brill, 2015, pp. 94-149. Sulle influenze filosofiche orientali v. in particolare E. GARIN, Il platonismo e la dignità dell'uomo, in: IDEM, Umanesimo italiano, Bari, Laterza \& Figli, 1973, pp. 106-132.

8 M. Ficino, Opera omnia, Basileae, ex officina Henricpetrina, 1576, p. 1836.

9 P. CASTelli, I geroglifici..., cit., p. 8.
} 
sapienti, che ora trasmettono il loro messaggio all'occidentale cultura quattrocentesca e, in particolare, conferma l'interesse per la sacra scrittura del sapiente Egitto, ricollegabile alla ricerca della lingua universale dell'umanità degli intellettuali tesi a conciliare le tradizioni antiche e bibliche. Nelle immagini geroglifiche, destinate esclusivamente agli "iniziati", si vedeva una trasmissione della scienza esoterica della potenza dei simboli "non detti" che solo gli dei possono intendere, ${ }^{10}$ e ora è Polifilo a misurarsi con i contenuti espressi non solo dalla scrittura pittografica, ma anche dall'intero oggetto che trova sul suo cammino.

Le opere egizie, riportate in primo piano dopo la scoperta di Horapollone diventarono esempi da prendere a modello per i soggetti delle storie e per i monumenti, lo erano anche i ritrovamenti romani e greci. La piramide con l'obelisco è il primo edificio che Colonna descrive a lungo e dettagliatamente con l'intenzione di ideare una costruzione remota, precisamente misurata e satura di un grandioso mistero. È una costruzione primigenia che con la sua immensità sovrasta ogni opera umana:

Imperò che questa amplissima structura sencia fallo excede la insolentia Aegyptica. Supera gli meravegliosi labyrinthi. Lemno quiesca. Theatri sa mutiscano, non si aequa el dignificato Mausoleo. Perché questo certamente non fue inteso da colui, che gli septe miracoli, overo spectacoli del mondo scripse. Né unque in alcuno saeculo, né viso, né excogitato tale, silendo etiam el sepulchro mirabile di Nino. ${ }^{11}$

Il progetto della struttura ibrida presentata a pagina $\mathrm{b} v$ attinge ai capolavori dell'architettura elencati dal Colonna e oltre all'ispirazione egiziana pare chiara, come osserva Hülsen, l'influenza dei miracoli dell'antica Grecia, specie quella del "dignificato Mausoleo":

[...] il disegno polifiliano non è altro che un tentativo di ricostruzione di uno dei più nobili monumenti greci, conosciuto agli umanisti del Quattrocento soltanto dalla letteratura antica $[\ldots]$ cioè il mausoleo di Alicarnasso, annoverato già nell'antichità fra

10 Jamblico, De mysteriis Aegyptiorum, II, 11, 96-97. Cfr. J. Sokolski, Wstęp, in: HorapolLON, Hieroglify, tr. J. KroczaK, Wrocław, W.U.W., 2003, p. 11.

11 HP b iii $v$. 
i sette miracoli del mondo, e descritto con grande esattezza da Plinio il giuniore nel libro XXXVI della Naturalis Historia (30-31). ${ }^{12}$

Fra le prove di una ricostruzione del mausoleo di Alicarnasso ${ }^{13}$, di particolare interesse, secondo la descrizione pliniana, sarebbe il disegno attribuito a uno dei probabili cooperatori per la parte artistica dell'Hypnerotomachia, Giovanni Monsignori, detto fra Giocondo da Verona. ${ }^{14}$

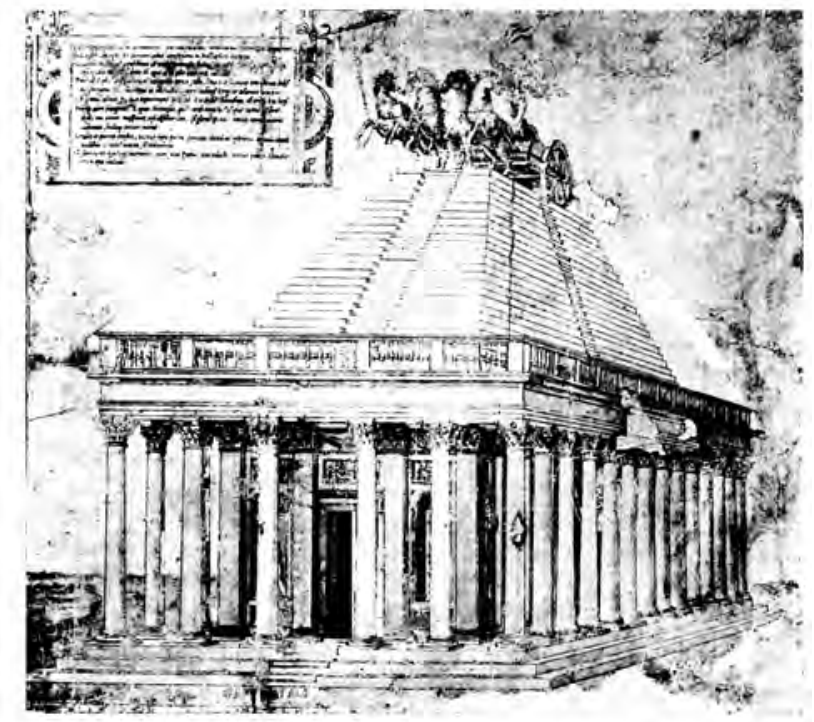

Fig. 1. Fra Giovanni Giocondo da Verona (1433 ca. - 1515): Ricostruzione del mausoleo di Alicarnasso. Firenze, Galleria Uffizi, il. foglio Uffizi 240.

"Il basamento quadrato con l'ornato di colonne, la piramide a gradini si trovano anche qui; soltanto in cima, conforme alla descrizione pliniana, vi

\footnotetext{
12 Ch. Hülsen, Le illustrazioni della Hypnerotomachia Poliphili e le antichità di Roma, estratto dal vol. XII, anno XII, dispensa 5-66 della "Bibliofilia", Firenze, Olschki, 1910, pp. 9, 11. 13 Ivi, p. 9.

14 H.A. Geymüller, Cento disegni di architettura d’ornato e di figure di Frà Giovanni Giocondo, Firenze, Bocca, 1882, p. 37. L'ipotesi di una cooperazione per la parte artistica dell'Hypnerotomachia di Fra Giovanni Giocondo da Verona, che nel 1511 pubblicò a Venezia la prima edizione illustrata del De Architectura di Vitruvio, avanzata da Domenico Gnoli, è negata da CH. HüLSEN, Le illustrazioni..., cit., pp. 9-13.
} 
è la quadriga colla statua del Re, invece dell'obelisco polifiliano" - rimarca Hülsen. ${ }^{15}$ Comunque sia, la composizione polifilesca rappresentata da dettagli che "l'arte antica non avrebbe mai congiunti insieme"16 è un invito a una reinterpretazione delle idee simboleggiate da ogni particolare del complesso che parla di una cultura universale. L'obelisco posto sopra la piramide costituisce un novum rispetto all'immagine di Plinio e anche se i due oggetti sono congiunti e formano l'unica struttura, rimangono ben identificabili come oggetti singoli, e ognuno di essi è carico di significati metaforici e filosofici.

Per il percorso spirituale di Polifilo il ruolo dei caratteri egizi si riduce in generale a quello delle "chiavi per penetrare nella dimensione misterica" che fungono da ambiguo tramite alla conoscenza. ${ }^{17} \mathrm{La}$ piramide che incontra Polifilo è decorata di scritte in diverse lingue, messe l'una accanto all'altra, e tale disposizione indica il legame tra culture diverse, ma accomunate dall'arte, dal pensiero e dalla scrittura. Della prima epigrafe incisa sull'obelisco della piramide (b ii verso) non si ha nel testo una citazione esplicita e diretta, sappiamo, tuttavia, che alla base della costruzione raffigurata è impiombata una targa di bronzo che dice: "Sotto poscia della prona piana del Obelisco, una tabella aenea era implumbata resupina, cum antiqua scriptura de notule nostrate, de Graece et Arabe, per le quale pienamente io compresi, al summo Sole quello dedicato". ${ }^{18}$ L'obelisco è quindi dedicato al Sole, similmente agli obelischi reali che simboleggiavano il dio del sole Ra che si diceva fosse un raggio di sole pietrificato dell'aten, il disco solare. ${ }^{19}$ L'iscrizione è nella lingua "nostra", accompagnata da quella greca e araba, argomento su cui si tornerà più avanti.

La lunga descrizione della struttura piramidale data dal Colonna, si sofferma su due oggetti: la scultura di bronzo di un cavallo alato con dei putti romani in groppa e la statua - già menzionata - di un gigantesco elefante su cui si leva un obelisco con dei geroglifici incisi e ambedue possono essere citate come esempio di trasgressione dei confini dello spazio, delle culture e del tempo. La rappresentazione dell'elefante polifilesco conduce alle idee del

\footnotetext{
15 CH. HüLSEN, Le illustrazioni..., cit., p. 11.

16 Ivi, p. 9.

17 O. Pelosi, Cinque saggi sul Polifilo, Salerno, Edisud, 1987, p. 17.

18 HP b viii $r$.

19 Qui da notare la tradizione pliniana che parla di "obeliscos vocantes Solis sacratos. Radiorum eius argumentum in effigie est". Cfr. F. ColonNA, ed. cit., vol. II, p. 537.
} 
Physiologus, testo creato al fine di sostenere i cristiani egiziani nella comprensione della natura secondo i principi della nuova religione che ormai si affermava nei territori dell'Impero romano. Sulla scia della scuola alessandrina, il mondo vi è percepito come un riflesso della realtà spirituale e immateriale, mentre la natura è interpretata secondo le idee della religione cristiana. Così, l'immagine complessiva del creato e la concezione delle cose dell'universo si esprimono nelle raffigurazioni di una continua lotta tra il bene e il male, del conflitto tra il positivo e il negativo e gli stessi principi del cosmo e dei suoi elementi sono visti in un incessante processo di compenetrazione e di fusione. Le descrizioni simboliche di animali e di piante reali e immaginarie contenute nel testo, lette in chiave allegorica attraverso citazioni delle Scritture, si riferiscono ai significati metafisici che descrivono il cosmo e i diritti umani. Secondo il Fisiologo, gli elefanti sono effigi dei primi genitori che quando abitavano nell'Eden non conoscevano l'unione carnale, come gli elefanti che non desiderano congiungimento sensuale. Si diceva, poi, che non avessero articolazioni alle ginocchia, per cui quando cadevano e non erano più in grado di rialzarsi si mettevano a barrire chiedendo aiuto. In loro soccorso arrivavano dodici altri elefanti, ma nessuno riusciva a rialzarlo:

Così arrivò il grande elefante, cioè la Legge, e non poté portarlo, poi arrivarono i dodici elefanti o un gruppo di profeti, ma non riuscirono neppure a sollevare un uomo caduto, dopo che tutti loro un santo elefante di spirito venne e sollevò l'uomo dalla terra. Il più grande di tutti divenne schiavo di tutti: si umiliò, assumendo la forma di uno schiavo, per salvare tutti. Dopo di tutti è arrivato il santo elefante spirituale e ha sollevato l'uomo da terra. Il più grande di tutti è divenuto schiavo di tutti: ha umiliato se stesso per salvare tutti. ${ }^{20}$

Con il Fisiologo l'elefante entra nell'immaginario letterario-religioso come simbolo della Legge e sostegno dell'uomo caduto a causa del peccato originale. La scultura dell'elefante nell'Hypnerotomachia se racchiude in sé significati tramandati da diverse tradizioni orientali e occidentali (e non è questo il luogo in cui ci vogliamo soffermare), si presta alle interpretazioni delle idee e del sapere di varie culture e zone in contatto. Il cubo di pietra su cui

20 Il Fisiologo, a cura di F. ZАмBon, Milano, Adelphi, 1975, passus 43. 
è posta la statua riprodotta nell'illustrazione $\mathrm{b}$ vii $v$ dal lato mostrato nella silografia del libro è coperto di scritti geroglifici, l'altro lato invece, nascosto nell'illustrazione, è coperto da scritte che dovrebbero chiarire il senso delle epigrafi geroglifiche. Anche le scritture geroglifiche dell'obelisco posto sul dorso dell'elefante sono accompagnate da iscrizioni latine, greche e arabe: vi si trova una specie di sella con un pettorale sul quale sono scritte le parole: "cerebrum est in capite"; sulla fronte dell'animale è posta, invece, una lamina con le parole in arabo e in latino:

Dalla quale uno ambitioso ornato, summamente notabile di eramento traiectato per sopra il suo amplissimo fronte pendeva, di dui quadrati composito, cum liniamenti elegante. Nella planitie dil quale (di foliatura undiculare circundata) vidi alcune littere Ionice, et Arabe le quale cusi diceuano. ${ }^{21}$

Con ciò Colonna riconferma che la lingua e la scrittura araba acquistano un posto accanto alla lingua latina e greca: ed è una conquista filologica degli intellettuali quattrocenteschi che ampliano i loro studi e indirizzano le ricerche verso il mondo e le tradizioni orientali. ${ }^{22}$

Le iscrizioni arabe che cita il testo di Polifilo sono tre: la prima è riportata sul foglio b ii $v$, la seconda sul foglio b vii $r$, e la terza sul foglio $h$ viii $r$. La prima delle epigrafi non è illustrata, le altre due sono accompagnate dalle rispettive silografie. Le iscrizioni silografate hanno un'estrema importanza non tanto per lo stesso libro, ma per la storia della lingua araba, in quanto costituiscono il primo testo arabo pubblicato a stampa nel mondo ${ }^{23}$. Il libro

\footnotetext{
$21 \quad H P$ b vii $r$.

22 Il fenomeno assume un'importanza particolare quando Giovanni Pico della Mirandola esprime il progetto di una conciliazione degli scritti testamentari contrastanti, ma collazionabili, e Marsilio Ficino propone un confronto dottrinale fra le religioni giudaica, cristiana e musulmana. Sulla medesima linea di riflessione insisteva ancora De ente et uno di Giovanni Pico della Mirandola dell'anno 1491 con l'idea di ricostruire i tratti essenziali di una filosofia universale, che guidi alla concordia fra diverse correnti di pensiero sorte sin dall'antichità che, accomunate dalla sapienza e dall'aspirazione al divino, culminano nel messaggio cristiano della Rivelazione. M. Ficino, De christiana religione, Firenze, 1474 e G. Pico della Mirandola, De ente et uno, 1492, recentemente pubblicato in: De ente et uno e scritti vari, a cura di E. GARIN, Torino, Nino Aragno, 2004.

23 M.A. Piemontese, Le iscrizioni arabe nella «Poliphili Hypnerotomachia», in: Ch. BurnettA. Contadini, Islam and the Italian Renaissance, London, Warburg Institute, 1999, p. 207.
} 
non cita epigrafi arabe a sé stanti, tutte si presentano accompagnate da un'altra lingua. Per questo pare significativa la scelta delle lingue delle iscrizioni che sono citate in latino, in greco e in arabo la prima; in greco e in arabo la seconda, e in greco, latino, ebreo ed arabo la terza.

Le altre due epigrafi arabe sono riportate in contesti testuali diversi, anche se la prima di esse, la sopracitata ( $b$ vii $r$ ), rimane nell'ambito della stessa struttura figurativa dell'obelisco. Come menzionato, questi tracciati arabi sono illustrati in silografie: l'epigrafe (b vii $r$ ) si trova incisa sulla figura dell'elefante, sulla fronte dell'animale che fa parte della struttura piramidale, dove è posta una lamina con un testo bilingue, in greco e in arabo.

La scia epigrafica conduce all'interno dell'elefante; dentro ci sono due figure incoronate e nude, poste su due sarcofagi: nella parte posteriore è presente la figura di un uomo che con la mano destra tiene uno scettro rivolto verso il davanti e con la mano sinistra si appoggia su uno scudo a forma di teschio di cavallo. Qui si leggono parole in tre lingue: ebraico, greco e latino. Così il greco e il latino rimangono la base stabile di comunicazione e di trasferimento dei contenuti culturali, mentre al posto dell'arabo è presente la lingua ebraica. Il significato delle iscrizioni rimane inspiegato, ma chi segue attentamente la narrazione arriva al momento del racconto in cui la ninfa Logistica rivela a Polifilo che la chiave per chiarire il senso dei messaggi si trova proprio sulla testa dell'elefante, che è l'elemento che indica il dito della donna. ${ }^{24}$ Vi è incisa una piastra di metallo con una sentenza bilingue, questa volta in greco e in arabo:

Adverti che sopra del suo fronte depende l'ornato cum quella ancipite descriptione, la quale in materno et plebeo sermone dice. Fatica et industria. Imperoché nel mundo chi vivendo vole thesoro havere, lassi stare el marcescente ocio, significato per il corpatio, et togli la decorata testa, che è quella scriptura et harai thesoro affaticantise cum industria. ${ }^{25}$

Secondo le spiegazioni della ninfa, la figura maschile racchiusa nell'elefante rappresenta il corpo e l'ozio, la figura femminile, invece, l'intrapren-

\footnotetext{
24 F. Colonna, ed. cit., vol. II, p. 146.

25 HP h vi $v$.
} 
denza e la comprensione. Qui si va verso la sfera teologica e spirituale: è un'esortazione a superare la dimensione fisica e a formare una mente forte, per avere il "thesoro", ovvero la saggezza divina e l'emanazione della luce dall'uno, simboleggiate dall'obelisco. La scelta dei due idiomi e l'accostamento fra greco e arabo indicano la complementarità del sapere scientifico e filosofico comunicato attraverso le rispettive lingue e culture. ${ }^{26} \mathrm{Si}$ tratta di un possibile parallelismo tra pensiero sapienziale occidentale e orientale, espresso attraverso l'idea umanistica di conciliazione delle filosofie e delle visioni del mondo contrastanti, ma integrabili, se inserite in un contesto nuovo, che non sia riletto ed interpretato secondo rigide norme dell'uno o dell'altro sistema filosofico-culturale. Il senso italiano corrisponde al significato greco, ma non è corrispettivo all'arabo che si legge come "fatica e conoscenza" o "opera e conosci". Tuttavia, in entrambe le versioni, dette parole possono essere intese come una variante del motto benedettino ora et labora e della dantesca idea di "virtude e conoscenza". ${ }^{27}$

Fino ad ora, il significato di fines viene emblematizzato da distinte lingue di diverse zone temporali e culturali poste l'una accanto all'altra; il confine viene cancellato proprio mediante l'accostamento di vari idiomi tesi a esprimere un'idea simile; diverse lingue messe insieme simboleggiano l'unione di culture che si complimentano e trasmettono la stessa connessione: virtus-labor, ossia l'idea della fatica intellettuale che porta alla conoscenza. Il concetto è illustrato in una delle scene nodali dell'Hypnerotomachia che rappresenta il momento della scelta della via che deve compiere Polifilo, trovandosi davanti alle tre grandi porte, ornate dalle iscrizioni "di charactere Ionico, Romano, Hebraeo, et Arabo" che portano alla Cosmodoxia (Gloria del Mondo), quella a destra, alla Theodoxia (Gloria di Dio), quella a sinistra, e alla Erototrophos (Madre d'Amore), la porta centrale (h viii $r$ ). La scena parla della "giusta scelta" della via nella vita, ma in questa scelta c'è posto per la cultura unita da un linguaggio comune, espresso dall'accostamento delle lingue, fino ad allora mai sperimentato. Pare significativa la scelta delle lingue delle iscrizioni che sono citate in latino, in greco e in arabo la prima; in greco e in arabo la seconda, e in greco, latino, ebreo ed arabo la terza. Le lingue ivi riportate sono le tre

26 M.A. Piemontese, Le iscrizioni arabe..., cit., p. 207.

$27 \quad$ Ivi, p. 208. 
lingue sacre per eccellenza: l'ebraico, il greco e il latino, ${ }^{28}$ cui se ne aggiunge una quarta: l'arabo.

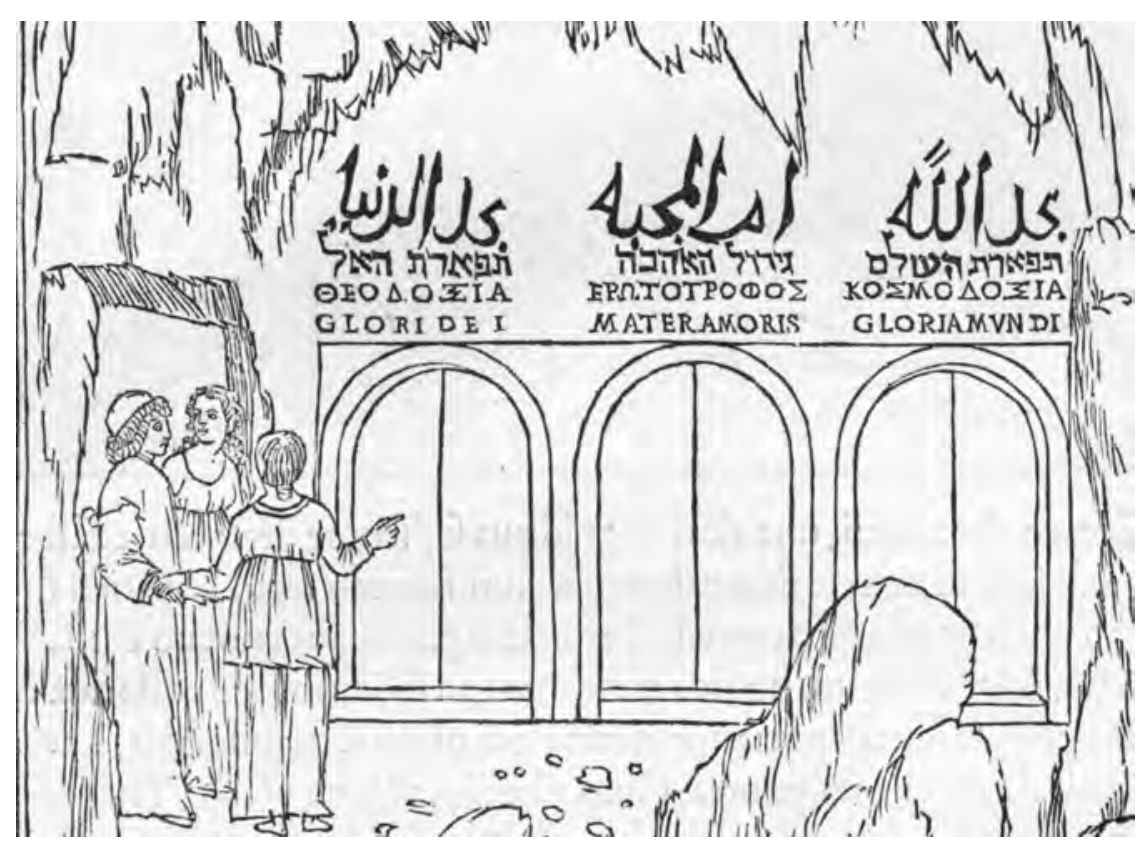

Fig. 2. HP h viii $r$ (dettaglio). ${ }^{29}$

Qui le lettere arabe sono disegnate da qualcuno che, con ogni probabilità, non conosceva la lingua e aveva soltanto copiato i caratteri, tuttavia i caratteri grafici sono chiari e ben leggibili: con virtuosismo vi si espone la calligrafia che è l'arte islamica per eccellenza. L'accostamento di diverse lingue rappresenta, di nuovo, un consenso universale fra le dottrine di diverse tradizioni, quella cristiana e quella musulmana: qui si arriva a un'espressione dell'identità, della stessa condizione umana in lontani ambiti spaziali e temporali, dove l’importanza della giusta scelta è decisiva per la sorte universale dell'uomo. ${ }^{30}$

\footnotetext{
28 Di tali lingue sacre parla IsIdoro DI SivigLia nelle Etymologiae: IX, 1, 5.

29 Le illustrazioni dell'Hypnerotomachia riportate nel presente testo provengono dall'edizione dell'Hypnerotomachia Poliphili del 1499 che si conserva a Venezia nella Nuova Manica Lunga, Fondazione Cini, FOAN TES 171.

30 Cfr. A. Klimkiewicz, Cultura sincretica dell'Hypnerotomachia Poliphili di Francesco Colonna, "Cuadernos de Filologia Italiana”, vol. XXI, 2014, pp. 190-191.
} 
Il confine e il limite sono significativi espedienti narrativi che permettono di scoprire e di ordinare il sistema umano del sapere del mondo terreno e del cosmo: qui, alla base, vi sono la ragione, la comprensione e la conoscenza delle leggi che regolano la natura. Il confine che consente un contatto con diversi mondi: l'interno e l'esterno, il reale e l'irreale è rilevante nell'ekphrasis di opere architettoniche, sculture e rilievi, ma prima di tutto nelle rappresentazioni dei giardini. L'uscita dal complesso piramidale segna, infatti, il fine di una tappa del viaggio dominata dalla successione di stati interni e dinamici e l'inizio di una nuova che porta verso un isolato "mondo-giardino", mondo vegetale, in cui Polifilo subirà la trasformazione della "rinascita". Qui lo spazio è ragionevolmente ordinato e regolato da aree e zone artificialmente chiuse da confini che sono determinati dalla natura e dalle sue forme specifiche. Le linee che separano i singoli territori, sempre carichi di significato simbolico, sono ben evidenziate, la loro funzione è quella di sottolineare la geometrizzazione dei terreni giardinieri.

Una tappa sostanziale del viaggio onirico di Polifilo si svolge nella leggendaria terra di Afrodite, dea dell'Amore, e altrettanto leggendaria città ideale di carattere umanistico-rinascimentale vista come un sistema di giardini. Colonna nel suo progetto giardiniero è profondamente suggestionato dalla simbolica figura polivalente del cerchio che racchiude in sé infinite possibilità, dalla raffigurazione dell'Assoluto alla conciliazione delle religioni rivelate, concedendo di descrivere l'esistenza dell'uomo come microcosmo e la sua superiorità sulle altre creature. Con il cerchio si esprimono le idee portanti della visione umanistica del cosmo: la corrispondenza dei corpi celesti agli esseri terrestri, l’influsso dei pianeti sulle vicende terrene, la ciclicità delle stagioni correlata ai ritmi delle età umane e le dottrine della magia teorica e operativa. ${ }^{31}$ Con un continuo ricorso alla geometria del cerchio si elabora un modello dell'universo appoggiato sulla centralità e sul limite, oppure la loro mancanza; il concetto trova il suo culmine nella forma dell'isola di Citera, nella composizione delle sue parti e nel "miraveglioso templo" di Venere, il cui disegno e il centro di costruzione sono appoggiati sul raggio geometrico. ${ }^{32}$

Le descrizioni architettoniche di cui abbonda il testo si riferiscono al sapere trasmesso da Vitruvio e Alberti, ma Colonna sottolinea particolarmente

31 V. anche O. Pelosi, Cinque saggi..., cit., pp. 18-20.

32 Par. la silografia in: HP n iii $r$. V. anche la Fig. 1 nel testo di Schwab-Laue in questo volume. 
la relazione concettuale e simbolica tra la circolare struttura del tempio di Venere e l'immagine della dea "genetrice feconda e materna". Il concetto deriva dal pensiero albertiano che aveva unito la forma del cerchio all'idea dell' "universale generazione naturale". ${ }^{33}$ Anche lo stesso progetto del tempio impostato sul cerchio, con una cupola e una galleria-chiostro è ispirato al pensiero di Alberti. ${ }^{34}$ La gigantesca struttura archeologico-architettonica, simile a costruzioni quattrocentesche, rinvia alla tarda antichità e all'era paleocristiana, vi sono ben chiari i riferimenti sia al De re adificatoria albertiano che al De architectura vitruviano, ma la stessa descrizione del palazzo data dal Colonna rimane un'inventio prevalentemente letteraria che sembra avere poco in comune con gli edifici antichi, esistenti all'epoca.

Terminata la tappa dei giardini e passata la sosta nel tempio di Venere, Polifilo si dirige, verso il "destructo tempio" e le zone dominate da un remoto ricordo dell'antichità, rappresentate dal polyandrion e racchiuse in forma di oggetti che appartengono alla sfera della morte. Per ritornare alla tipologia delle illustrazioni a piena pagina nel libro osserviamo che la seconda parte di tali illustrazioni ed esattamente la metà (sei su dodici) delle figure elencate in precedenza è dedicata al trapasso nell'altro mondo e allude alla fine della vita umana. Così, vi si trovano due antichi epitaffi ( $\mathrm{q} v v$, q viii $r$ ), tre sepolcri: quello di Artemisia ( $\mathrm{r}$ iii $r$ ), un sepolcro con due busti ( $\mathrm{r}$ ii $r$ ) e un terzo con un'arca con due porticine ( $\mathrm{r}$ iiii $r$ ) e, alla fine, il doppio epitaffio dedicato a Polia (F iii v). Tutto sommato, tre grandi costruzioni tombali e tre grandi epitaffi, collocati a poca distanza di tre, quattro e una pagina.

Una chiave di lettura della parte finale del racconto diventa il topos dell'umana fragilità, ${ }^{35}$ in quanto il romanzo si chiude con la morte di Polia ed è illustrato da un'iscrizione tombale. L'epitaffio, che si intitola Epitaphium ubi Polia loquitur, contiene un'epigrafe latina che potrebbe chiarire il senso generale

33 L.B. Alberti, De re cedificatoria, a cura di A. Poliziano, Strasbourg, Jakob Kammerlander, 1541, pp. 549-550.

34 V.L. Donati, Polifilo a Roma. Il mausoleo di S. Costanza, "La Bibliofilia", LXX, 1968, pp. 1-38; M. Calvesi, La Pugna d'amore in sogno, Lithos, Roma, 1996, p. 86; G. Pozzi-M.T. CASElla, Francesco Colonna. Biografia e opere, Padova, Antenore, 1959, t. I, p. 156 ss.

35 F. Colonna, ed. cit., vol. II, pp. 932-933. 


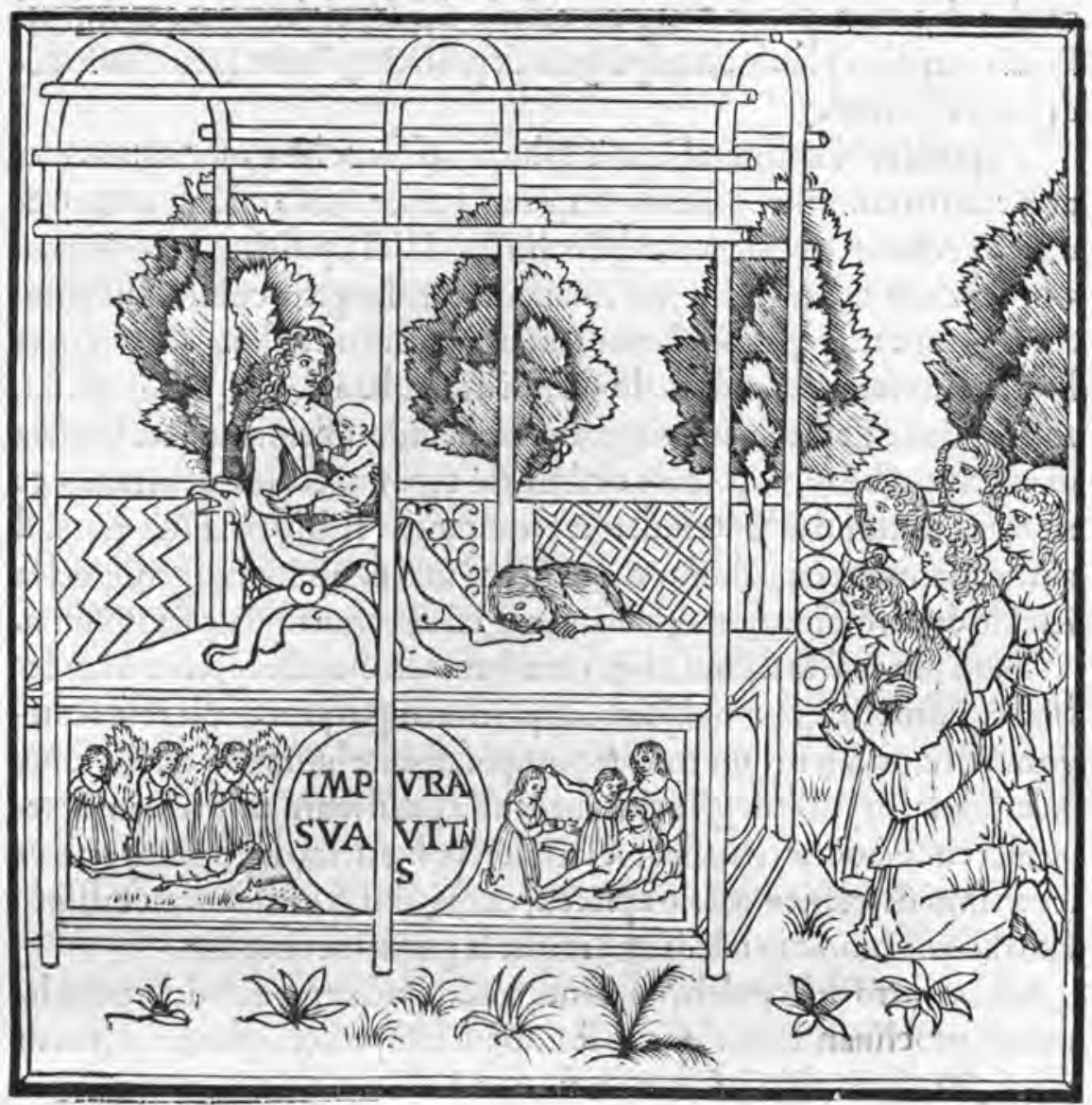

Fig. 3. HP z viii r: Sancta Venere Divina Genitrice.

del libro - è l'ipotesi avanzata da Paolo Cortesi ${ }^{36}$ che, comunque sia, illustra il nostro tema e la proposta di interpretare la struttura dell'opera con l'uso di concetti relativi al sincretismo, espresso dalla trasgressione di limes. L'irregolare disposizione grafica delle linee dell'epitaffio di Polia ha incitato il Cortesi a sottoporre il testo a numerose analisi, disponendone le singole lettere su griglie e applicando griglie sia numeriche che alfabetiche e alla fine, alla sistemazione delle singole lettere dell'epigrafe in modo che l'ultima lettera di ogni riga fosse incolonnata a destra. ${ }^{37}$ In risultato è stato quello di aver individuato

36 P. CoRTesi, Manoscritti segreti. Dai misteri del Mar Morto alle profezie di Nostradamus, Roma, Newton \& Compton, 2003, p. 93 ss.

$37 \quad$ Ivi, pp. 93-94. 


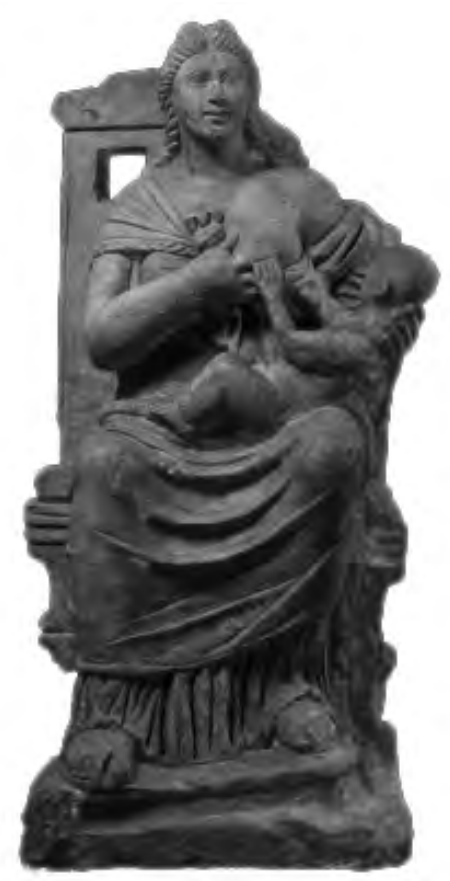

Fig. 4. Isis lactans (Egitto, arte copta, IV-V d.C. ca.), Staatliche Museen zu Berlin, Skulpturensammlung und Museum fuer Byzantinische Kunst.

una sequenza di dodicesime lettere a partire dal margine destro che formano una catena di segni che si leggono come: VOSELECIEISEOVVII. La sequenza contiene le lettere maiuscole che celano la proposizione latina: VOS ELECI E ISEO V V I I A, il cui significato si legge come: Vos eleci e Iseo Venus Victrix Invicta Immortalis Aeterna. Sono le parole che a Polifilo e Polia rivolge la stessa Venere, per dire: "Voi scelsi dal tempio di Iside. Venere vincitrice invitta immortale eterna". La dea dell'Amore si mostra qui come un'immortale vincitrice, in cui rinascono la natura e il genere umano, pari alla Vergine Maria e, come testimonia la lettura del messaggio nascosto tra i segni dell'epigrafe, pari a Iside. Così, la figura principale dell'Hypnerotomachia, la dea Venere si presenta in unione con la dea egizia e con la Madre di Dio cristiana. Per poter mettere il segno di uguaglianza tra Iside e Venere si ricorda il passo che conclude la prima parte del romanzo, comprendente la scena in cui i due protagonisti vanno sacrificati alla dea durante una suggestiva cerimonia, in cui Venere si identifica con la dea egizia. Con l'immagine del rinato figlio di Venere, rappresentata in una scena che fortemente allude al mito della rinascita di 
dio egizio Osiride, risuscitato da Iside, si determina l'ambiente di culto isiaco in cui culmina l'iniziatico percorso di Polifilo (HP z viii $r$ ).

Il capolavoro di Colonna, che si riferisce a molte tradizioni letterarie e culturali, sebbene non appartenga chiaramente a nessuna precedente, non diventa un modello per altre opere, sebbene molte ne traggano ispirazione. Le complicate idee ivi espresse formano un complesso sistema di conoscenza, mai ripetuto in una simile forma letteraria. Con l'Hypnerotomachia si parla dell'unione dell'uomo con l'universo, accomunati dalle stesse eterne regole dell'esistenza e dallo stesso desiderio di "sapientia" e si crea un racconto del mondo e sul mondo della rinascita aperto alle utopie della comunicazione universale, convergenti nell'idea di costruzione dello spazio di cultura in comune, per superare le limitazioni geografico-temporali della conoscenza umana. 